\title{
Avaliação do aplicativo "Gestação" na perspectiva da semiótica: o olhar das gestantes
}

\author{
Evaluation of the 'Gestação' application from the perspective \\ of semiotics: pregnant women's views
}

Francisca Francisete de Sousa Nunes Queiroz (http://orcid.org/0000-0002-2933-4974) ${ }^{1}$

Christina César Praça Brasil (https://orcid.org/0000-0002-7741-5349) ${ }^{1}$

Raimunda Magalhães da Silva (http://orcid.org/0000-0001-5353-7520) ${ }^{1}$

Indara Cavalcante Bezerra (http://orcid.org/0000-0003-0647-2490) ${ }^{1}$

Patrícia Moreira Costa Collares (http://orcid.org/0000-0002-1048-0330) ${ }^{2}$

José Eurico de Vasconcelos Filho (http://orcid.org/0000-0002-6881-0814) ${ }^{3}$

${ }^{1}$ Programa de Pós-

Graduação em Saúde

Coletiva, Universidade de Fortaleza (Unifor). Av.

Washington Soares 1321,

Edson Queiroz. 60811-905

Fortaleza CE Brasil.

zete.queiroz@hotmail.com

${ }^{2}$ Centro de Ciências da

Saúde, Universidade Federal

do Ceará. Fortaleza CE

Brasil.

${ }^{3}$ Núcleo de Aplicação em

Tecnologia da Informação,

Unifor. Fortaleza CE Brasil.
Abstract Evaluating health-related applications is necessary for their adequacy and dissemination in society. The GestAção application was evaluated based on the opinion of 13 pregnant women followed up in prenatal care consultations at three public health services and at a private institution in Fortaleza, Ceará, Brazil. Data emerged from an evaluation carried out from June to December 2017, including semi-structured interviews. Data were analyzed from content analysis in thematic and semiotic modality. Thus, the theme "meanings attributed to the application by pregnant women" emerged, which is associated with the following cores of meaning: provision of safe and diverse information; possibility of changing behavior and motivations for using the application. It is considered that semiotics allowed to understand the meanings attributed to the application from the interaction of pregnant women with the tool. The GestAção application was considered satisfactory, motivating to use, informative, safe and promoter of changes in the context of gestational health. Participants' views allow the evaluated technology to compose strategies aimed at promoting health care for pregnant women.

Key words Pregnancy, Biomedical technology, Mobile applications, Prenatal care, Woman's health
Resumo A avaliação de aplicativos voltados à saúde é necessária para a sua adequação e disseminação na sociedade. O aplicativo GestAção foi avaliado com base na opinião de 13 gestantes acompanhadas em consultas pré-natais, em três serviços públicos de saúde e em uma instituição privada, em Fortaleza, Ceará, Brasil. Os dados emergiram do processo avaliativo realizado de junho a dezembro de 2017, incluindo entrevistas semiestruturadas, tendo sido analisados a partir da análise de conteúdo na modalidade temática e da Semiótica, resultando no tema "significados atribuídos ao aplicativo pelas gestantes", a qual se associa aos seguintes núcleos de sentido: fornecimento de informações seguras e diversificadas; possibilidade de mudança de comportamento e motivações para a utilização do aplicativo. Considera-se que a Semiótica permitiu compreender os significados atribuídos ao aplicativo, a partir da interação das gestantes com a ferramenta, indicando o GestAção como satisfatório, motivador ao uso, informativo, seguro e promotor de mudanças no contexto da saúde gestacional. O olhar das participantes possibilita que a tecnologia avaliada possa compor as estratégias voltadas à promoção $e$ aos cuidados à saúde das gestantes.

Palavras-chave Gravidez, Tecnologia biomédica, Aplicativos móveis, Cuidado pré-natal, Saúde da mulher 


\section{Introdução}

A gestação é um período de significativa importância para a mulher, permeado por dúvidas, temores e curiosidades ante as mudanças corporais e psíquicas que acompanham essa fase ${ }^{1}$. Motivadas pela necessidade de obter conhecimentos, seja pela inexperiência ou pelo desejo de compartilhar suas vivências com outras pessoas, as mulheres buscam em sites, aplicativos (app) e redes sociais informações claras e seguras sobre a gestação atual ou futuraa ${ }^{2-4}$.

O uso da tecnologia móvel propicia a obtenção de informações relacionadas à saúde devido à acessibilidade, disponibilidade e conectividade instantâneas ${ }^{5,6}$. A rápida expansão da internet abriu espaço para diversas áreas, dentre elas, a saúde eletrônica (eHealth), a qual promove uso acessível e seguro de tecnologias de informação e comunicação para suporte à saúde e campos afins, incluindo o cuidado, a vigilância, a educação em saúde, o ensino e a pesquisa ${ }^{7}$. Quando o ambiente virtual de saúde é amparado por dispositivos móveis, como telefones celulares, dispositivos de monitoramento de pacientes, entre outras ferramentas sem fio, denomina-se mobile Health (mHealth) ${ }^{7}$.

Tais ferramentas têm fortalecido os serviços e a disseminação do conhecimento em saúde por permitirem a incorporação do aconselhamento profissional e o compartilhamento de informações seguras. Nesse domínio, os smartphones revolucionaram a forma das pessoas comunicarem-se, compartilharem e consumirem conteúdos $^{8}$.

Assim, diversos aplicativos estão sendo utilizados para auxílio clínico, melhoria da educação continuada e da assistência ao paciente, contribuindo para a promoção da comunicação em saúde ${ }^{9}$. Estudos relatam os benefícios do uso de aplicativos em intervenções em saúde, no que diz respeito à melhoria da tomada de decisões clínicas, educação de pacientes e profissionais da saú$\mathrm{de}^{9,10}$. Desse modo, desenvolver soluções no formato de aplicativos representa um meio eficaz de atingir o público-alvo. Entretanto, outras pesquisas demonstram que a maior preocupação para a utilização dessas ferramentas na área da saúde é a falta geral de regulação e de evidências ${ }^{11,12}$.

Nesse contexto, o aplicativo GestAção foi concebido e desenvolvido por uma equipe interdisciplinar da Universidade de Fortaleza, em 2014. Trata-se de uma ferramenta tecnológica voltada ao empoderamento da gestante, contendo informações multimídia e de fácil compreensão sobre as fases do período gestacional. Os conteúdos são individualizados, contextualizados culturalmente e acessíveis ao público-alvo. Ademais, conta com um inquérito de saúde voltado ao registro de dados sobre o pré-natal, mediante um sistema especialista capaz de identificar gestantes em situações de risco, apoiando o direcionamento das ações de pré-natal ${ }^{13}$.

Com efeito, o app GestAção constitui um suporte informativo, por meio do qual as mulheres podem esclarecer dúvidas e acompanhar o processo gestacional de forma interativa, podendo contribuir para o agendamento de consultas no pré-natal e servir de guia para as ações recomendadas no período gravídico-puerperal, uma vez que sinaliza a necessidade de exames (laboratoriais e imaginológicos), consultas, vacinas, a data provável do parto, entre outras informações relevantes ${ }^{13}$.

Para que essa tecnologia possa ser amplamente transferida para os serviços de saúde e disseminada para o uso de gestantes e profissionais, é necessário que seja avaliada e validada quanto à segurança e clareza das informações, manuseio, aparência, funcionalidade, entre outras características. Avaliar esse tipo de tecnologia se faz necessário, visto que estas ferramentas trazem implicações na vida das pessoas, levam os usuários a mudanças de atitudes e comportamentos, além de potencializarem o cuidado em favor da qualidade de vida e da saúde.

O estudo objetivou avaliar o aplicativo GestAção, a partir do olhar de gestantes, à luz da Semiótica.

\section{Método}

O estudo faz parte de um projeto intitulado " $m$ Health para promoção de saúde da mulher: inovação tecnológica para melhoria da qualidade da assistência pré-natal", financiado pela Fundação Cearense de Apoio ao Desenvolvimento Científico e Tecnológico (FUNCAP). Os resultados desse projeto vêm sendo desdobrados em diversas produções científicas, a exemplo de artigos, livros e capítulos ${ }^{13,14}$.

A investigação é de natureza aplicada, metodológica, com abordagem qualitativa, voltada à validação ampla de um recurso tecnológico, $\mathrm{o}$ aplicativo GestAção, por meio da opinião de gestantes em acompanhamento pré-natal.

Para a avaliação de tecnologias, dentre outras técnicas, sugere-se o processo de validação por especialistas e pelo público-alvo ${ }^{15}$. Com efeito, 
a validação de tecnologias implica nas formas mais diretas de Interação Humano-Computador (IHC) e a capacidade de um artefato provocar mudanças atitudinais e/ou comportamentais em seus usuários ${ }^{16}$. Esta fase do estudo compreendeu a validação do aplicativo GestAção, valendo-se da Semiótica, enquanto ciência que associa o significado dado à interação entre o usuário e a tecnologia em questão.

A semiologia, no campo da linguagem, relaciona-se à ciência que estuda os fenômenos de produção social dos sentidos. Numa cultura, todos os objetos são revestidos de sentido, com os significantes interagindo culturalmente com as pessoas, formando uma cadeia de interpretantes mediante um ciclo infinito de produção de sentidos e efeitos ${ }^{17}$.

Portanto, a Teoria Semiótica estuda os signos - unidades de sentido e significação que uma pessoa atribui a algo, a alguém ou a alguma situação. A Semiótica procura entender como as pessoas interpretam as coisas e os fatos no contexto que as envolvem, considerando que o sujeito atribui significado a tudo o que o rodeia ${ }^{18}$. Compreender como as pessoas interagem com os objetos, como elas pensam e se emocionam são algumas das possibilidades que a Semiótica oferece.

Embora ainda seja pouco explorado pela metodologia científica no campo da saúde, a Semiótica favorece o estudo das compreensões e mudanças de atitude decorrentes da Interação Humano-Computador, sendo respaldada por várias teorias da comunicação ${ }^{16,18,19}$. Nesse contexto, considerou-se esta teoria para fundamentar a validação do aplicativo GestAção pelas mulheres grávidas no âmbito da assistência pré-natal ${ }^{18}$.

A etapa avaliativa realizou-se de junho a dezembro de 2017, incluindo três momentos. No primeiro momento, houve a seleção de 13 gestantes que estavam realizando consulta pré-natal, as quais foram abordadas nas salas de espera de duas Unidades de Atenção Primária à Saúde (UAPS); no Programa de Atenção à Gestante da Universidade de Fortaleza (UNIFOR) e na Maternidade Escola Assis Chateaubriand (MEAC), na cidade de Fortaleza, Ceará, Brasil.

Os critérios de inclusão no estudo foram gestantes com as seguintes características: disponibilidade para participar dos encontros para treinamento sobre o manuseio do aplicativo; mulheres com período gravídico até o segundo trimestre gestacional; em acompanhamento prénatal; que possuíssem smartphones com tecnologia Android; e com facilidade de acesso à internet no seu dia a dia (via $W i-F i, 3 G, 4 G$ ). Os critérios de exclusão corresponderam a: mulheres com gravidez de risco, além de alguns outros motivos relacionados aos seus smartphones - pacote de dados reduzido, velocidade de conexão lenta, acesso Wi-Fi limitado e troca do número do telefone.

Sobre as participantes, verificou-se que a idade das gestantes variou de 13 a 35 anos, sendo que seis delas eram adolescentes (com idade inferior a 18 anos de idade); todas tinham baixo nível de escolaridade (apenas duas concluíram o ensino médio); cinco viviam maritalmente com um parceiro, enquanto oito estavam solteiras; e nove não possuíam nenhuma renda. Dez gestantes eram primigestas e 11 relataram não apresentar doenças crônicas. Todas as gestantes utilizavam aplicativos em seus celulares, bem como navegavam nas redes sociais. Entretanto, chama a atenção, neste estudo, o fato de nove participantes não conhecerem nenhum aplicativo sobre gestação.

No segundo momento, ocorreu um treinamento individual com as gestantes guiado pela equipe de pesquisa acerca do tutorial do aplicativo, fornecendo explicações sobre seu manuseio. Em seguida, o aplicativo GestAção foi instalado nos respectivos smartphones, ficando à disposição para uso por um período que variou de um a três meses. Ressalta-se que, durante esse tempo, as gestantes foram acompanhadas quinzenalmente pela equipe de pesquisa.

A última parte da investigação em campo ocorreu de novembro a dezembro de 2017, quando finalizou o período de utilização do aplicativo pelas gestantes. Nessa oportunidade, foi realizada uma entrevista semiestruturada com cada participante, utilizando-se um roteiro baseado nas premissas da Semiótica, para captar as suas experiências de uso com o GestAção. As perguntas disparadoras foram: do que você mais gostou no aplicativo GestAção? Quais contribuições o aplicativo trouxe para seu processo gestacional? Você teria sugestões para aprimorar o GestAção? Se sim, quais?

Com base nas gravações das entrevistas e anotações dos pesquisadores, os dados foram transcritos e organizados a partir da Análise de Conteúdo na modalidade temática ${ }^{20}$, evidenciando-se núcleos de sentido e suas ideias associadas. A partir dessa análise, emergiram os sentidos e os significados atribuídos pelas gestantes ao aplicativo GestAção. Ressalta-se que o amparo à interpretação dos dados respaldou-se na Semiótica ${ }^{21}$, buscando a compreensão de como as gestantes se apropriam e significam sua interação com a tecnologia em destaque, possibilitando verificar 
como vêm à tona as ideias, as representações e as respectivas motivações relacionadas ao GestAção.

Para proteger as identidades das participantes, adotaram-se letras e números. Assim, G1 refere-se à “Gestante 1", e assim, sucessivamente.

Os preceitos ético-legais da pesquisa seguiram as recomendações da Resolução no 466, de 12 de dezembro de 2012, do Conselho Nacional de Saúde, a qual aponta as Diretrizes e Normas Regulamentadoras de Pesquisa Envolvendo Seres Humanos $^{22}$. O projeto foi aprovado pelo Comitê de Ética da Universidade de Fortaleza.

\section{Resultados}

Diante da análise dos dados fundamentados na Semiótica, emergiu das entrevistas a temática "significados atribuídos ao aplicativo pelas gestantes", considerada como o assunto mais abrangente e significativo para a verificação das percepções das gestantes sobre o aplicativo GestAção e os seus efeitos no processo gestacional. Para sistematizar e organizar os resultados analíticos, optou-se por apresentar três Núcleos de Sentidos (NS), os quais convergem opiniões e alinham ideias, que respaldam a sua significação.

O primeiro NS refere-se às "informações seguras e diversificadas" oferecidas pelo aplicativo, o que destaca aspectos relacionados à compreensão das mulheres sobre o seu papel informacional e aborda elementos que versam sobre a influência da ferramenta para a busca e a aquisição de conhecimentos. O segundo NS corresponde à "possibilidade de mudança de comportamento", o qual volta-se para a tomada de consciência das mulheres sobre a necessidade de cuidado e a valorização do aplicativo como instrumento interativo e de apoio à gestação. Por último, as "motivações para a utilização do aplicativo" apresenta signos que levam à compreensão sobre a adesão das gestantes ao uso da ferramenta.

\section{Informações seguras e diversificadas}

Ao fazerem referências às informações que o aplicativo GestAção traz, destaca-se, nas opiniões das participantes deste estudo, a credibilidade atribuída à ferramenta. Observa-se que o aplicativo se configurou, durante o período de uso, como um apoio informacional sobre a gestação, estabelecendo uma relação de confiança no processo de IHC, como expresso nos relatos:

[...] ser mãe pela primeira vez foi bom, mas tendo o aplicativo GestAção foi melhor, porque é algo que informa a gente [...], tipo, algo que eu tinha dúvida, eu tirava as minhas dúvidas nele (no aplicativo). (G6)

[...] o aplicativo, porque tem todos os temas, ele explica bem direitinho, é seguro. Então foi ótimo para mim. (G12)

[...] eu gostei porque me ajudou a aprender mais sobre a minha gravidez. Gostei porque ele dá mais acesso às informações confiáveis que nem todo mundo tem. (G4)

Para essas gestantes, as informações seguras e advindas de fontes confiáveis inspiram credibilidade, o que denota a importância desses aspectos para a escolha da via de informação que causa interesse e que será utilizado pelo público-alvo. Os discursos revelam que o aplicativo GestAção proporcionou uma experiência que remete à confiança em relação às informações apresentadas. Não obstante, no relato de G4, observa-se a hesitação da gestante quanto à confiança nos conteúdos acessados em outras fontes.

\section{Possibilidade de mudança de comportamento}

As gestantes revelaram a atualização de conhecimentos após uso do GestAção. Ressaltaram pontos que julgam importantes, os quais resultam em atitudes saudáveis durante a gravidez, significando que o novo conhecimento levou a atitudes e mudanças positivas em seu cotidiano. Nesse contexto, os aplicativos têm um grande potencial na promoção da saúde, tornando os usuários mais conscientes e interessados em suas condições de vida. Ressalta-se também que a maioria dessas ferramentas tecnológicas tem um papel importante para que as pessoas adotem estilos de vida mais saudáveis, conforme demonstrado nos relatos a seguir:

[...] a minha alimentação mudou muito, não tinha uma alimentação saudável e mudei bastante depois que eu fui saber o que podia acontecer e o que eu precisaria para mudar. $O$ aplicativo mostra tudo isso e de uma forma bem fácil! (G6)

Tenho uma amiga que está no comecinho da gravidez. Eu falei para ela do aplicativo que estou usando [...]. Como não vai ser toda hora que ela poderá falar comigo para tirar as suas dúvidas, eu vou ter sempre uma resposta para ela, mas ela tendo o aplicativo GestAção para acessar, vai ser mais fácil ela aprender a se cuidar melhor. (G5)

Com base nos relatos, percebe-se que o aplicativo é capaz de disseminar uma variedade de informações impactantes acerca do cuidado na 
gravidez, promovendo a corresponsabilização das gestantes acerca do processo gestacional.

\section{Motivações para a utilização do aplicativo}

O app GestAção revela um potencial motivacional e organizativo, por ser uma ferramenta que agrega aspectos informativos e interativos, proporcionando às gestantes ferramentas para realizar seu planejamento diário e individual, organizar e agendar consultas e compromissos, além de ser inclusivo, permitindo o acesso à informação às pessoas que não sabem ler.

[...] as minhas vitaminas, às vezes, eu esquecia [...], aí eu olhava o aplicativo, lembrava e tomava. Ele (o aplicativo) também me ajudou bastante porque eu sou muito nova e o aplicativo me ajudou a ver o que eu não sabia. (G4)

[...] gostei do aplicativo porque, se eu sentir alguma coisa, eu posso anotar lá para não esquecer e para quando eu for a uma consulta falar com a médica. (G5)

Eu gostei da rádio que tem no aplicativo porque mostra bem direitinho como é que tem que ser quando o bebê nasce. Eu achei mais interessante ainda essa rádio porque tem muitas mãezinhas que não sabem ler, escrever, entendeu? A rádio ajuda essas pessoas e também tem muita coisa na rádio que é explicada de uma forma mais resumida. (G10)

Fica evidente que o aplicativo detém aspectos que causam motivação de uso e de mudanças atitudinais nas usuárias, sobretudo por ser de fácil manuseio e autoexplicativo.

A facilidade de uso e o que ele (o aplicativo) explica, a facilidade de ver (encontrar as informações), de manusear, porque o que havia nesse aplicativo, a forma de manusear, não tinha no outro que eu usei. (G6)

Tive experiência com um aplicativo que eu não lembro o nome. Só que esse tal aplicativo que eu tive, que foi através de uma colega minha, ele não tinha todos os conteúdos que esse aplicativo aqui tem (referindo-se ao GestAção) e nem as explicações da alimentação, por exemplo, não tinha. $O$ que tinha no outro aplicativo eram explicações sobre o período de gestação; mas informações sobre alimentação e medicamentos não tinha. É tanto que aquele aplicativo eu nem passei a usar, logo eu tirei, porque ele não me motivou. O GestAção é diferente, gosto muito do que ele oferece e isso motiva, né? (G12)

As falas revelam alguns motivos que estimularam o uso do aplicativo GestAção pelas participantes, trazendo indicativos de uma avaliação positiva sobre a facilidade de acessar, de manusear e de localizar informações, além da diversidade de informações contidas.

\section{Discussão}

As tecnologias em saúde são efetivas para atender as necessidades dos usuários e geram impactos positivos $^{2,11,23-27}$. Os aplicativos surgiram como meios de mediação e provimento de informações, por possuírem grande potencial comunicativo e de educação em saúde, além de auxiliarem na promoção da saúde e do bem-estar ${ }^{28}$.

O aplicativo GestAção é um desses exemplos, visto que favorece o aprendizado sobre os cuidados necessários para uma gravidez saudável. Entretanto, não se pode esquecer da importância do enfoque avaliativo, já que esse é um processo capaz de aferir o potencial de uma ferramenta tecnológica e a importância dos atributos que ela traz, apresentando-se como etapa indispensável no processo de utilização ampla de aplicativos ${ }^{29}$. Com efeito, há necessidade de se avaliar tecnologias desenvolvidas para a área da saúde, o que inclui a validação da eficácia e da confiabilidade desses recursos antes da utilização da ferramenta com uma população maior ${ }^{12,23}$. Nessa perspectiva, o conhecimento das experiências do público -alvo e os efeitos decorrentes da utilização de um aplicativo é um dos aspectos mais importantes no processo de validação desse tipo de ferramenta ${ }^{14}$.

As participantes deste estudo faziam uso de diversos aplicativos em seus smartphones, entretanto, algumas delas relataram não conhecer nenhum aplicativo sobre gestação. Este fato diverge do estudo de McKay et al. ${ }^{26}$, o qual evidenciou que a maioria das gestantes faz download, numa média de três aplicativos contendo informações sobre a gravidez, durante o período gestacional.

No tocante à diversidade de conteúdo e segurança do app, o GestAção configurou para as gestantes como uma ferramenta confiável de apoio informativo. Barbosa e Silva ${ }^{30}$ reconhecem que esses aspectos estabelecem uma relação de confiança no processo de Interação Humano-Computador (IHC). Nesse cenário, as mulheres estão cada vez mais interessadas em novos conhecimentos que lhes tragam estratégias apoiadoras e baseadas em evidências científicas ${ }^{14}$.

$\mathrm{Na}$ perspectiva da Semiótica ${ }^{18}$, os significados atribuídos ao aplicativo pelas participantes do estudo remete a uma experiência de confiança e credibilidade, ficando evidente o potencial da ferramenta. As gestantes expressaram senti- 
mentos de satisfação com o uso do aplicativo e a possibilidade de mudança de comportamento, revelando seu potencial como promotor de transformações atitudinais por meio do conteúdo e da qualidade de informações oferecidas.

Nesse sentido, estudos ${ }^{8,11,16,23}$ referem que o diálogo da ferramenta com o usuário deve promover a mudança de comportamento em favor da saúde e que as aplicações em informática na saúde podem fazer diferença na vida das pessoas, pois servem como aliados a uma série de conteúdos e serviços, estimulando a promoção de comportamentos mais saudáveis. Com efeito, uma linguagem bem direcionada e clara favorece ao usuário a compreensão e a formulação ou ressignificação de sentidos, o que possibilita a adoção de condutas que melhorem suas condições de saúde ${ }^{23}$.

Estudo $^{27}$ que investigou intervenções respaldadas pelo uso de aplicativos demonstrou a capacidade desses dispositivos em incentivar mudanças de comportamentos relativos às atividades físicas e dietéticas. Delgado et al. ${ }^{23}$ afirmam que a utilização de algumas estratégias e ferramentas mobile leva a mudanças ou adequações comportamentais que facilitam a promoção da saúde.

Quanto às motivações para a utilização do aplicativo, as gestantes apontaram aspectos que estimulam o uso do GestAção, revelando suas percepções sobre os impactos positivos do aplicativo na incorporação de conhecimento sobre o tema. Confirmaram, ainda, que as recomendações propostas para a melhora do estilo de vida oferecidas pelo app trazem estímulos motivacionais e efeitos positivos, evidenciando que isto impulsiona o uso da ferramenta.

Estudos confirmam ${ }^{12,24}$, em vários aspectos, a eficácia do uso de aplicativos para a autogestão de práticas de saúde e chama a atenção para a necessidade de elementos motivadores que gerem maior adesão dos participantes. Nesse sentido, esta investigação evidenciou aspectos que causam motivação quanto ao uso do GestAção pelas gestantes que avaliaram positivamente a facilidade de acessar, de manusear e de localizar informações, além da diversidade de informações que oferece ao público-alvo. Verifica-se, portanto, que este aplicativo fornece importantes contribuições para melhorar as intervenções e estratégias voltadas à saúde materno-infantil.

Diante do exposto, os signos revelados pelas experiências das gestantes quanto ao uso do Ges-
tAção, ampliam sua utilidade para os serviços de saúde e os profissionais que podem lançar mão dessa ferramenta tecnológica como potencializadora do cuidado ao pré-natal. Com efeito, o aplicativo avaliado mostrou-se funcional, adequado ao uso, com características motivadoras e eficazes para o objetivo ao qual se propõe.

O estudo revela uma contribuição importante na área da saúde, pois certifica, por meio da avaliação do público-alvo, a utilização segura e eficaz de uma ferramenta tecnológica voltada ao cuidado pré-natal. Ademais, pode servir de exemplo para outros estudos avaliativos, na perspectiva qualitativa, pois refere à utilização de um método analítico fundamentado na Teoria Semiótica, o qual ainda é pouco utilizado na área da saúde, mas que se mostra adequado para estudos avaliativos de tecnologias $m$-health e sua interação com humanos.

As limitações da investigação decorrem das dificuldades na captação das participantes devido à falta de acesso à internet de algumas gestantes interceptadas nas instituições que acolheram o estudo; e a falta manutenção de contato com as participantes por motivos de mudança do número do telefone, roubo de celular, entre outras razões.

\section{Considerações finais}

A avaliação do aplicativo GestAção, sob o olhar das gestantes, revelou ser este uma tecnologia facilitadora e coadjuvante ao cuidado materno-infantil no período gestacional, por possibilitar o processo interativo, educativo, contínuo, acessível e seguro da mulher grávida às informações e ações de saúde requeridas nesta fase.

As participantes atribuíram ao aplicativo sob avaliação, significados que remetem à motivação de uso, capacidade de transformações atitudinais e de apoio à gravidez, o que incentiva à corresponsabilização das mulheres nos cuidados com a saúde, na melhoria das suas atitudes e no fortalecimento da autonomia e do autocuidado das gestantes.

A avaliação do aplicativo GestAção foi positiva, na opinião das usuárias, indicando que esta tecnologia pode ser disseminada para a sociedade como uma ferramenta tecnológica que auxilia o cuidado com a saúde durante o pré-natal. 


\section{Colaboradores}

FFSN Queiroz, CCP Brasil, RM Silva, IC Bezerra, PMC Collares e JE Vasconcelos Filho participaram em todas as etapas do estudo, perpassando pelo planejamento, revisão de literatura, coleta e análise de dados, escrita do artigo e revisão crítica do material.

\section{Referências}

1. Collares PMC. Inovação tecnológica da assistência prénatal [Tese]. Fortaleza: Universidade de Fortaleza; 2014.

2. Calderon TM, Cestari MEW, Dobkowski AC, Cavalheiro MD. The use of the Internet as a support tool to clarify questions during pregnancy. Health Biol Sci 2016; 4(1):18-22.

3. Frazer C, Hussey L, Bosch E, Squire M. Pregnancy App: A Closer look at the implications for child bird educators. International Journal of Childbirth Education 2015; 30(3)

4. Hoga LAK, Reberte LM. Pesquisa-ação como estratégia para desenvolver grupo de gestantes: a percepção dos participantes. Rev Esc Enferm USP, 2007; 41(4):559-566.

5. Lee Y, Moon M. Utilization and Content Evaluation of Mobile Applications for Pregnancy, Birth, and Child Care. Healthc Inform Res 2016; 22(2):73-80.

6. Kraschnewski JL, Chuang CH, Pool ES, Peyton T, Blubaugh I, Pauli J, Feher A, Reddy M. Paging "Dr. Google": Does technology fill the gap created by the prenatal care visit structure? Qualitative focus group study with pregnant women. J Med Internet Res 2014; 16(6).

7. World Health Organization (WHO). mHealth: New horizons for health through mobile technologies: second global survey on eHealth. Genebra: WHO; 2012.

8. Meyer RN. M-health: aplicativo para celular para materno-infantil. 2016. In: Mostra interna de trabalhos de iniciação científica, 7. Maringá. Anais. Maringá: UNICESUMAR.

9. Souza JF, Goncalves FB, Queiroz VAR, Queiroz RS. Avaliação de um aplicativo para auxílio à tomada de decisão de mobilizar pacientes críticos. Rev. Saúde Com 2015; 11(1):59- 68.

10. Costa SRS, Duqueviz BC, Pedroza RLS. Tecnologias Digitais como instrumentos mediadores de aprendizagem dos nativos digitais. Rev. Quadrimestral da Associação Brasileira de Psicologia Escolar e Educacional 2015; 19(3).

11. Kayyali R., Peletidi A, Ismail M, Hashim Z, Bandeira P, Bonnah J. Awareness and Use of mHealth Apps: A Study from England. Pharmacy: Journal of Pharmacy, Education and Practice 2017; 5(2):33.

12. Rocha TAH, Fachini LA, Thumé E, Silva NC, Barbosa, ACQ, Carmo, M. Saúde Móvel: novas perspectivas para a oferta de serviços em saúde. Epidemiol. Serv. Saúde 2017; 25(1):159-170.

13. Silva RM, Brasil CCP, Bezerra IC, Queiroz FFSN. Mobile health technology for gestational care: evaluation of the GestAção's App. Rev Bras Enferm 2019; 72(Supl. 3):266-273.

14. Queiroz FFSN, Brasil CCP, Silva RM, Collares PMC, Vasconcelos Filho JE. Os significados atribuídos pelas gestantes ao aplicativo GestAção. Investigação Qualitativa em Saúde - CIAIQ Atas 2018; 2.

15. Pereira FGF, Frota NM, Silva DV, Sousa LMO, Almeida JC, Cysne Filho FMS. Avaliação de aplicativo digital para o ensino de sinais vitais. Rev Min Enferm - Reme 2017; 21. 
16. Onodi L, Costa I, Gadelha, B. Universia: Um Aplicativo Colaborativo de Suporte a Grupos Acadêmicos. Anais do XXVII Simpósio Brasileiro de Informática na Educação - SBIE; 2016; Manaus, Amazonas. [acessado 2019 Nov 26]. Disponível em: http://dx.doi. org/10.5753/cbie.sbie.2016.1245

17. Pinto MJ. As marcas linguísticas da enunciação: esboço de uma gramática enunciativa do Português. Rio de Janeiro: Numen Ed.; 1994.

18. Santaella L, Noth W. Introdução a semiótica: Passo a passo para compreender os signos e a significação. São Paulo: Paulus; 2017.

19. Salvador PTCO, Mariz CMS, Vítor AF, Ferreira Júnior MA, Fernandes MID, Martins JCA, Santos VEP. Validation of virtual learning object to support the teaching of nursing care systematization. Rev Bras Enferm 2018; 71(1):11-19.

20. Minayo MCS, Deslandes SF. Caminhos do pensamento: Epistemologia e Método. Rio de Janeiro: Fiocruz; 2013.

21. Santaella L. Semiótica Aplicada. São Paulo: Cengage Learning; 2008.

22. Brasil. Conselho Nacional de Saúde. Resolução no 466, de 12 de dezembro de 2012. Diretrizes e normas regulamentadoras de pesquisas envolvendo seres humanos. Diário Oficial da União 2013; 13 dez.

23. Delgado M, Miranda S, Rodrigues PF. Uma avaliação das aplicações mobile classificadas em saúde e fitness. Acta Port Nutr 2017; 8:22-26.

24. Jo IY, Yoo SH, Lee DY, Park CY, Kim EM. Diabetes Management via a Mobile Application: a Case Report. Clin Nutr Res 2017; 6(1):61-67.

25. Calderon T M, Cestari MEW, Dobkowski AC, Cavalheiro MD. The use of the Internet as a support tool to clarify questions during pregnancy. J Health Biol Sci 2016; 4(1):18-22.

26. McKay FH, Cheng C, Wright A, Shill J, Stephens H, Uccellini M. Evaluating mobile phone applications for health behaviour change: A systematic review. Journal of Telemedicine and Telecare 2018; 24(1):22-30

27. Yang $\mathrm{CH}$, Maher JP, Conroy DE. Implementation of Behavior Change Techniques in Mobile Applications for Physical Activity. Elsevier 2015; 48(4):452-455.

28. Carvajal DN, Brittner MR, Rubin SE. Can apps reduce rates of teen pregnancy? J Fam Pract. 2015; 62(10):538-598.

29. Santos L. Teste e avaliação de usabilidade de app para gestão de diabetes em Android [dissertação] Porto: Faculdade de Ciências da Universidade do Porto em Ciências de Computadores; 2015.

30. Barbosa SDJ, Silva BS. Interação humano-computador. Rio de Janeiro: Elsevier; 2011.

Artigo apresentado em 11/03/2020

Aprovado em 27/04/2020

Versão final apresentada em 29/04/2020

Editores chefes: Maria Cecília de Souza Minayo, Romeu Gomes, Antônio Augusto Moura da Silva 